\title{
Multi-objective metaheuristic algorithms for the resource-constrained project scheduling problem with precedence relations
}

\author{
Helton Cristiano Gomes ${ }^{a, *}$, Francisco de Assis das Neves ${ }^{a}$, \\ Marcone Jamilson Freitas Souza ${ }^{\mathrm{b}}$ \\ a Departamento de Engenharia Civil, Universidade Federal de Ouro Preto, Campus Universitário - Morro do Cruzeiro, Ouro Preto, \\ 35400-000 Minas Gerais, Brazil \\ ${ }^{\mathrm{b}}$ Departamento de Ciência da Computação, Universidade Federal de Ouro Preto, Campus Universitário - Morro do Cruzeiro, Ouro Preto, \\ 35400-000 Minas Gerais, Brazil
}

\section{A R T I C L E I N F O}

\section{Available online 12 November 2013}

Keywords:

Project management

Resource constrained project scheduling

Multi-objective optimization

Metaheuristics

\begin{abstract}
A B S T R A C T
This study addresses the resource-constrained project scheduling problem with precedence relations, and aims at minimizing two criteria: the makespan and the total weighted start time of the activities. To solve the problem, five multi-objective metaheuristic algorithms are analyzed, based on Multi-objective GRASP (MOG), Multi-objective Variable Neighborhood Search (MOVNS) and Pareto Iterated Local Search (PILS) methods. The proposed algorithms use strategies based on the concept of Pareto Dominance to search for solutions and determine the set of non-dominated solutions. The solutions obtained by the algorithms, from a set of instances adapted from the literature, are compared using four multi-objective performance measures: distance metrics, hypervolume indicator, epsilon metric and error ratio. The computational tests have indicated an algorithm based on MOVNS as the most efficient one, compared to the distance metrics; also, a combined feature of MOG and MOVNS appears to be superior compared to the hypervolume and epsilon metrics and one based on PILS compared to the error ratio. Statistical experiments have shown a significant difference between some proposed algorithms compared to the distance metrics, epsilon metric and error ratio. However, significant difference between the proposed algorithms with respect to hypervolume indicator was not observed.
\end{abstract}

(c) 2013 Elsevier Ltd. All rights reserved.

\section{Introduction}

Scheduling problems have been broadly studied in literature. Among those, the project scheduling (PSP) has been prominent. According to Oguz and Bala [1], the PSP is an important problem and it is challenging for those responsible for project management and for researchers in the related field. As said by the authors, one of the reasons for its importance is that it is a common problem in a great number of real situations of decision making, such as problems that originate in the project management of civil construction. The PSP is challenging, theoretically, for belonging to the class of NP-hard combinatorial optimization problems [2]. Thomas and Salhi [3], for example, state that the optimal solution of the PSP is hard to determine, especially for large-scale problems with resource and precedence constraints.

\footnotetext{
* Corresponding author. Postal address: 35 Bourbon S., Jardim Ouro Verde, Carmo do Paranaíba, 38840-000 Minas Gerais, Brazil. Tel.: +55 318748 1302,

+55 3135522244 (home), + 553438515658 (home), +55 3438559320 (work).

E-mail addresses: helton.gomes@gmail.com, hcrisgomes@yahoo.com.br (H.C. Gomes), fassis@em.ufop.br (F. de Assis das Neves), marcone@iceb.ufop.br (M.J.F. Souza).
}

Despite several authors like Slowinski [4], Martínez-Irano et al. [5] and Ballestín and Blanco [6] consider that the resolution of the PSP involve several and conflicting objectives, few studies have been developed using this approach. According to Ballestín and Blanco [6], the number of possible multi-objective formulations for the PSP is very large, due to the countless objectives found in literature. These can be combined in several forms, thus generating new problems. Among the objectives that project managers are most interested in, according to Ballestín and Blanco [6], we can emphasize the following:

- minimization of the project makespan;

- minimization of the project earliness or lateness;

- minimization of the total project costs;

- minimization of the resources availability costs;

- minimization of the total weighted start time of the activities;

- minimization of the number of tardy activities;

- maximization of the project net present value.

According to Martínez-Irano et al. [5], the multi-objective formulation of a problem is particularly important when the objectives 
are conflicting, i.e., when the objectives may be opposed to one another.

In this work, the PSP with resource and precedence constraints (RCPSPRP) is addressed as a multi-objective optimization problem. Two conflicting objectives are considered in the problem: the makespan minimization and the minimization of the total weighted start time of the activities.

Several multi-objective optimization methods can be found in literature to solve this class of problems. Such methods can be basically divided into two groups: the classic and the metaheuristic methods. The classic methods consist of transforming the objective function vector into a scalar objective function, as it is the case of the Weighted Criteria and the Global Criterion methods. In this case the problem is treated as a mono-objective problem. The metaheuristic methods use metaheuristics to generate and analyze several solutions, as well as to obtain a set of non-dominated solutions. Literature revisions about the multiobjective metaheuristic methods, as published by Jones et al. [7], show the Multi-objective Tabu Search (MOTS) [8], the Pareto Simulated Annealing (PSA) [9], the Non-dominated Sorting Genetic Algorithm II (NSGA-II) [10] and the Strength Pareto Evolutionary Algorithm II (SPEA-II) [11] as the most used. According to Ballestín and Blanco [6], there are still few works that propose efficient methods for solving the multi-objective RCPSPRP. Due to the computational complexity of the RCPSPRP, according to Thomas and Salhi [3], the metaheuristic methods appear as the best form to solve it.

According to Ballestín and Blanco [6], Slowinski [4] was the first author to explicitly represent the RCPSPRP as a multi-objective optimization problem. In the last years, some authors have addressed the RCPSPRP this way, as is the case of Viana and Sousa [12], Kazemi and Tavakkoli-Moghaddan [13], Abbasi et al. [14], Al-Fawzan and Haouari [15], Ballestín and Blanco [6] and Aboutalebi et al. [16].

Recently, new metaheuristic methods have arisen in literature. The main examples are the Multi-objective GRASP (MOG) [17], Multi-objective Variable Neighborhood Search (MOVNS) [18] and Pareto Iterated Local Search (PILS) [19]. Such methods have been applied successfully in several types of problems, as have reported in [20-24]. However, no article was found in literature using these new multi-objective metaheuristic methods to solve the RCPSPRP.

Due to the success of using these new methods, variations of the MOG, MOVNS and PILS are analyzed in this study to solve the RCPSPRP. For this, five algorithms were implemented: a MOG, a MOVNS, a MOG using VNS as local search, named GMOVNS, a MOVNS with an intensification procedure based on [24], named MOVNS_I, and a PILS. From our knowledge, in terms of algorithms, no work was found using VNS as local search for the MOG, as was done in the GMOVNS.

To assess the efficiency of the implemented algorithms, the results obtained through the use of instances adapted from literature were compared through four multi-objective performance measures: distance metrics, hypervolume indicator, epsilon metric, and error ratio. Statistic experiments were also carried out aiming at verifying, if there is a significant difference between the algorithms regarding the used performance measures.

The rest of this paper is organized as following: in Section 2 a literature review is presented. In Section 3 the characteristics of the problem addressed in this study are described and in Section 4 some concepts of the multi-objective optimization are presented. In Section 5 the aforementioned multi-objective metaheuristic algorithms are described, while in Section 6 the characteristics of the instances, as well as the performance measures used to assess and compare the algorithms, are laid out. In Section 6 the results of the conducted tests are presented and analyzed. The last section concludes the work.

\section{Literature review}

In this section, we briefly describe some important works that have researched the multi-objective RCPSPRP.

Slowinski [4] applied the multi-objective linear programming to solve the multi-mode RCPSPRP, allowing activities preemption. Renewable and non-renewable resources were considered. Makespan and costs minimization were choosing as objectives. In Slowinski's [4] approach, the decision-making is done before the search for solutions. For this, weights were assigned to objectives, which were grouped in a linear objective function. Thus, the decision maker can prioritize one of the objectives. However, this approach present an important disadvantage, the difficulty in defining adequate weights to the objectives. In all the proposed algorithms in our work, the decision-making is done after the search for solutions. That is, a set of candidate solutions (ideally the Pareto-optimal front or an approximation of it) is calculated by the algorithms and then the decision maker selects a solution among them. Also, goal programming and fuzzy logic applications to the multi-objective RCPSPRP were discussed by the author.

The PSA and MOTS algorithms were implemented by Viana and Sousa [12] to solve the multi-objective RCPSPRP. Three minimizing criteria were used: makespan, mean weighted lateness of activities and sum of the violation of resource availability. Due to the lack of multi-objective instances for the problem, adaptations of instances taken from PSPLib were used to test the algorithms. The PSPLib contains numerous mono-objective instances for the RCPSPRP, and adaptations were made to enable the application of multiobjective algorithms. The used instances are composed by 12,18 , 20 and 30 activities and 4 renewable resources. In our work were also made adaptations in instances taken from PSPLib for the application of the proposed algorithms. The average and maximum distance metrics were used to assess and compare the algorithms efficiency. Except for the instances group with 20 activities, the MOTS obtained better results for the used metrics.

Kazemi and Tavakkoli-Moghaddan [13] presented a mathematical model for the multi-objective RCPSPRP considering positive and negative cash flows. The maximization of net present value and makespan minimization were considered as objectives. Weights were assigned to objectives, creating a linear objective function, and one optimization software was used to solve the model. Due to the computational complexity of the RCPSPRP (NPhard), the use of optimization softwares restricts the tests to instances with small number of activities and resources. The model was tested using four small instances with 12 activities. Kazemi and Tavakkoli-Moghaddan [13] have proposed also the application of NSGA-II to solve the problem. Instances with 10,12, $14,16,18$ and 20 activities were used in the computational tests. The instances were taken from PSPLib and adapted to multiobjective optimization.

Abbasi et al. [14] studied the multi-objective RCPSPRP considering two objectives, makespan minimization and robustness maximization. The authors grouped the two objectives in a linear objective function, like in Slowinski [4] and Kazemi and TavakkoliMoghaddan [13]. Abbasi et al. [14] described the same difficulty in defining adequate weights to the objectives. However, to generate different solutions for large-scale problems, the Simulated Annealing metaheuristic was used. A numerical example with fifty activities and only one renewable resource was used to illustrate the method.

Al-Fawzan and Haouari [15] have studied the multi-objective RCPSPRP with two objectives, makespan minimization and robustness maximization. A MOTS has been proposed to solve the problem. In the proposed MOTS, the serial schedule generation scheme (S-SGS) was used to generate initial solutions, in the same way as in the algorithms proposed in our work. The difference regarding our work 
is that Al-Fawzan and Haouari [15] have not used priority rules. Eight algorithm variants were considered in the computational tests. Each variant presents a different configuration for three algorithm parameters (number of steps, neighborhood size and aggregation function). In the tests were used 480 instances with 30 activities and 4 renewable resources. Like in Viana and Sousa [12] and Kazemi and Tavakkoli-Moghaddan [13], the instances were taken from PSPLib and adapted. The distance metrics were used to assess and compare the efficiency of the variants.

Ballestín and Blanco [6] have presented theoretical and practical fundamentals of multi-objective optimization applied to the RCPSPRP. The authors described the relevance of the multiobjective formulation for the problem. They present several objective functions for the problem and a study regarding seven multi-objective performance measures and their disadvantages. Also, a comparison between the SPEA-II, NSGA-II and PSA was presented using the makespan and resources availability costs minimizations as objectives. The S-SGS and P-SGS (parallel schedule generation scheme) were used to generate initial solutions for the algorithms. Like in our work, Ballestín and Blanco [6] have used priority rules in the methods. The activities parameters used as priority rules were the release date, due date and duration. Instances with 120 activities and 4 renewable resources, taken from the PSPLib and adapted, were used to test the proposed algorithms. Ballestín and Blanco [6] presented results limiting the number of generated solutions in 5000, 10,000, 25,000 and 50,000 . In our work, the results are presented limiting the number of generated solutions in 5000. The average distance metric was used to assess and compare the algorithms efficiency.

Aboutalebi et al. [16] have presented two evolutionary algorithms, NSGA-II and Multi-objective Particle Swarm Optimization (MOPSO), for the bi-objective RCPSPRP. The adopted objectives were the minimization of the project makespan and the maximization of the net present value, which are two common objectives of this problem in the literature. Instances with 10,12 , 18,20 and 30 activities and 4 renewable resources were used in the computational experiments. The instances also were taken from PSPLib and adapted to the multi-objective optimization. The spacing and maximum spread metrics and the metric $C$ were used to assess and compare the algorithms efficiency. The computational results showed the superior performance of the NSGA-II with regard to the proposed metrics.

No article was found in literature using the MOG, MOVNS and PILS to solve the multi-objective RCPSPRP.

\section{Problem statement}

The RCPSPRP consists of, given a set $A=\{1, \ldots, n\}$, with $n$ activities, and, another $R=\{1, \ldots, m\}$, with $m$ renewable resources with predefined availabilities $B_{k}$, determining the start time of execution $\left(s_{i}\right)$ of each one of the $n$ activities, assuring that the resource level and the precedence relation are not violated. The execution of each activity $i \in A$ has a duration (processing time) pre-determined $p_{i}$, a weight $c_{i}$ and demand $b_{i k}$ units of each resource $k \in R$.

The precedence relations determine that some activities need to be conducted in a particular sequence; that is, an activity cannot start while its precedent activities have not been finished.

Two conflicting objectives have been considered in the formulation used for the problem, the makespan minimization $\left(f_{1}(s)\right)$ and the minimization of the total weighted start time of the activities $\left(f_{2}(s)\right)$. The values of $f_{1}(s)$ and $f_{2}(s)$ are given by Eqs. (1) and (2), where $n+1$ is an artificial activity $\left(p_{n+1}=c_{n+1}=0\right.$, $b_{n+1, k}=0 \forall k$ ) that represents the last one to be concluded and $s_{n+1}$ represents the project's finishing time.

$f_{1}(s)=\operatorname{Min} s_{n+1}$

$f_{2}(s)=\operatorname{Min} \sum_{i=1}^{n} \frac{c_{i}}{s_{i}}$

In problems related to RCPSPRP, the makespan minimization is the objective more found in literature. Due to concern on meeting deadlines, the project managers always seek to finalize the projects as soon as possible. The objective $f_{2}(s)$ represents a much-discussed issue in project management: worth making a larger investment to perform the activities as early as possible. The generated solutions by the algorithms will present different relations between the used objectives, helping the project managers in decision-making.

\section{Some definitions of multi-objective optimization}

For the best understanding of the developed algorithms the definition of some concepts of multi-objective optimization are primarily necessary.

(1) Definition 1 - Pareto Dominance

Given the feasible solutions $s$ and $s^{\prime}$, it is found:

(1) if $f_{k}(s) \leq f_{k}\left(s^{\prime}\right)$ for all $k=1,2, \ldots, l$ and $f_{j}(s)<f_{j}\left(s^{\prime}\right)$ for any $j, s$ will be a solution that dominates $s^{\prime}$;

(2) if $f_{k}\left(s^{\prime}\right) \leq f_{k}(s)$ for all $k=1,2, \ldots, l$ and $f_{j}\left(s^{\prime}\right)<f_{j}(s)$ for any $j, s$ will be a solution dominated by $s^{\prime}$;

(3) if $f_{j}(s)<f_{j}\left(s^{\prime}\right)$ for any $j$ e $f_{i}(s)>f_{i}\left(s^{\prime}\right)$ for any $i, s$ and $s^{\prime}$ are stated non-dominated or indifferent.

(2) Definition 2 - Pareto Optimality

A feasible solution $s$ is named Pareto-optimal (or efficient) if there is no other feasible solution $s^{\prime}$ suck that $s^{\prime}$ dominates $s$, that is, a solution $s^{\prime}$ such as $f_{k}\left(s^{\prime}\right) \leq f_{k}(s)$ for all $k=1,2, \ldots, l$ and $f_{j}\left(s^{\prime}\right)<f_{j}(s)$ for any $j$.

The set of all Pareto-optimal solutions is termed Paretooptimal front and as a result of the defined concepts, all the solutions that belong to the Pareto-optimal front are nondominated (indifferent).

In all the algorithms proposed in this work the criterion of the Pareto Dominance was used, as described in this section, to assess the solutions generated along with its iterations and to determine the set of non-dominated solutions, denoted by $D^{*}$, to be returned by the algorithms.

\section{Methodology}

In this section the multi-objective algorithms proposed to solve of the RCPSPRP are described. In the first three sub-sections the common components of the five algorithms are presented, such as the representation of a solution, the generation of an initial solution and the neighborhood structures.

\subsection{Representation of a solution}

A solution for the RCPSPRP is represented by a list $s=\left\{s_{1}, s_{2}, \ldots, s_{n}\right\}$, where $s_{i}$ indicates the start time of the execution of the activity $i$.

To illustrate, let us consider the instance given in Table 1 . The instance has ten activities (named from 1 to 10) and two renewable resources ( 1 and 2 ). The availabilities of the resources are, respectively, 5 and 3 units. In this table, for each activity $i$, the duration $p_{i}$, the weight $c_{i}$, the demand for the resources 1 and 2 $\left(b_{i 1}\right.$ and $\left.b_{i 2}\right)$ and the successors activities are presented. The instance presented in Table 1 is an adaptation of Koné et al. [25]. 
An example of a feasible solution, not necessarily optimal, for the presented instance is the list $s=\{15,1,22,30,9,10,4,10,27$, 14\}. The Gantt chart representing the described solution for the instance is presented in Fig. 1.

In the presented solution it is observed that the activity 2 is the first to be executed $\left(s_{2}=1\right)$ and the activity 4 is the last $\left(s_{4}=30\right)$. For this solution the objective functions values are: $f_{1}(s)=35$ and $f_{2}(s)=606.44$.

\subsection{Initial solution generation}

The proposed multi-objective algorithms start from an initial set of non-dominated solutions generated through a priority rule based scheduling heuristic. According to Kolisch [26], usually, this heuristic is composed of a priority rule and a schedule generation scheme for the determination of feasible sequencing.

For the generation of the initial set of non-dominated solutions the S-SGS proposed by Kelley [27] was used. In S-SGS, activities in an activity list $L$ are scheduled in the order in which they appear in $L$; they are scheduled at the earliest clock time at which the required resources become available. An activity list $L$ is a precedence feasible list of all activities of the given project [32]. If more than one activity can be assigned at a certain clock time, the activity to be scheduled is selected based on a priority rule. In the S-SGS used, three different types of priority rules were used as mentioned later:

(1) Lower duration: a solution $s$ is generated by sequencing activities in non-decreasing order of the value of its duration;

(2) Bigger number of successors activities: a solution $s$ is generated by sequencing activities in non-increasing order of its numbers of successors activities;

(3) Lower weight: a solution $s$ is generated by sequencing activities in non-decreasing order of the value of its weight.

\subsection{Neighborhood structures}

Local search methods usually use a neighborhood search to explore the space of feasible solutions of the addressed problem.

Table 1

Data for an instance with 10 activities.

\begin{tabular}{lrrrrrrrrrrr}
\hline Activities & 1 & 2 & 3 & 4 & 5 & 6 & 7 & 8 & 9 & 10 \\
\hline$p_{i}$ & 7 & 3 & 5 & 5 & 6 & 4 & 5 & 4 & 3 & 7 \\
$c_{i}$ & 200 & 300 & 500 & 100 & 600 & 200 & 500 & 300 & 300 & 200 \\
$b_{i 1}$ & 0 & 2 & 3 & 3 & 2 & 1 & 1 & 1 & 1 & 3 \\
$b_{i 2}$ & 2 & 1 & 3 & 2 & 1 & 0 & 3 & 1 & 1 & 1 \\
Successors & 3 & 6,7 & 4,9 & 11 & 1 & 1 & 5,8 & 10 & 4 & 9 \\
\hline
\end{tabular}

The methods begin with a solution $s$, and generate a neighborhood of this solution. Such neighborhood is obtained by applying simple changes on solution $s$.

The algorithms developed in this paper use two neighborhood structures: exchange and insertion. For a given solution (sequence) $s$, the neighborhood structures are described below:

(1) Exchange Neighborhood $\left(N_{1}(s)\right)$ : the neighbors of $s$ are generated by interchanging two activities in the sequence. The size of neighborhood $N_{1}(s)$ is $n(n-1) / 2$.

(2) Insertion Neighborhood $\left(N_{2}(s)\right)$ : the neighbors of $s$ are generated by inserting one activity in another position of the sequence. The size of neighborhood $N_{2}(s)$ is $(n-1)^{2}$.

By using the described two neighborhood structures, infeasible solutions can be generated due to resource constraints and precedence relations, but only the feasible solutions generated are considered and assessed by the algorithms.

\subsection{Multi-objective metaheuristic algorithms for the RCPSPRP}

\subsubsection{MOG algorithm}

The Multi-objective GRASP (MOG) is a multi-objective optimization algorithm based on the metaheuristic Greedy Randomized Adaptive Search Procedure (GRASP) proposed by Feo and Resende [28]. The MOG version proposed in this work, based on Reynolds and Iglesia [17], is presented in the Algorithm 1.

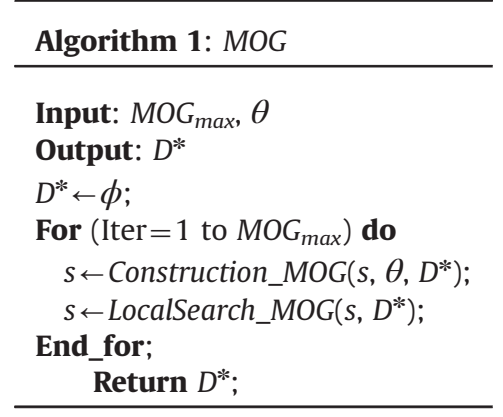

As in the method proposed by Feo and Resende [28], the $M O G$ is composed of two phases: construction and local search. In each one of the $M O G_{\max }$ iterations of Algorithm 1, a solution $s$ is generated in the construction phase through an adaptation of S-SGS. This adaptation consists of the insertion of a randomization rate $(\theta)$ to the method, being the greedy function, a characteristic of GRASP, based on the priority rules described in Section 5.2.

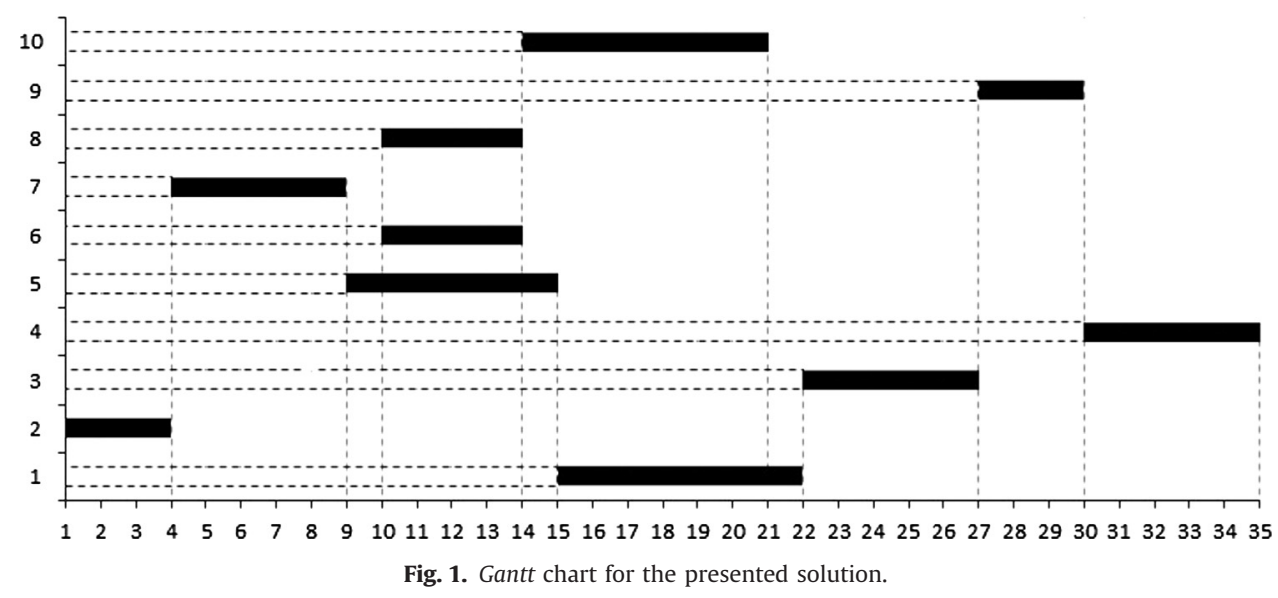


The pseudo-code of the procedure Construction_MOG is presented in Algorithm 1.1.

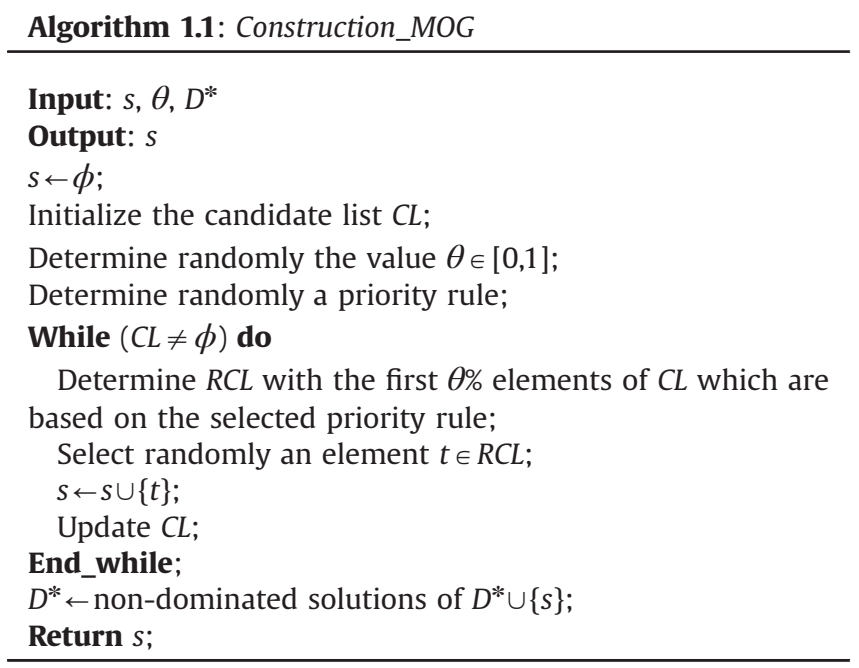

In Algorithm 1.1 the construction of a solution $s$ starts with the generation of a list of activities $C L$ that are candidates to be included in the sequencing. The $C L$ is determined by the available activities to the execution, at the time instant considered, and with its precedent activities already being sequenced. From the $C L$, the value of $\theta$, will define the restricted candidates list $(R C L)$, where the greedy function is determined by the priority rule selected in Section 5.2, that is, the activity that has the biggest priority will be the one that will bring the biggest benefit by being included in the sequencing. Once the $R C L$ is defined, an activity $t \in R C L$ is randomly selected and inserted in $s$, thus being the $C L$ updated. Finally, the solution $s$ generated is assessed to be part or not of $D^{*}$. Aiming at the generation of different solutions over the Pareto front, the value of $\theta \in[0,1]$ and the priority rule to be used are randomly determined by each Construction_MOG procedure call.

In the local search phase, the solution $s$ generated by the Algorithm 1.1 is modified by the exchange movement $\left(N_{1}(s)\right)$, described in Section 5.3, in a way that new solutions are generated. The pseudo-code of the procedure LocalSearch_MOG is presented in Algorithm 1.2.

\begin{tabular}{l} 
Algorithm 1.2: LocalSearch_MOG \\
\hline Input: $s, D^{*}$ \\
Output: $D^{*}$ \\
Determine randomly a neighbor solution $s^{\prime} \in N_{1}(s)$; \\
For $\left(\right.$ each neighbor $\left.s^{\prime \prime} \in N_{1}\left(s^{\prime}\right)\right)$ do \\
$\quad D^{*} \leftarrow$ non-dominated solutions of $D^{*} \cup\left\{s^{\prime \prime}\right\}$; \\
End_for; \\
Return $D^{*} ;$
\end{tabular}

The Algorithm 1.2 starts with a random determination of a solution $s^{\prime} \in N_{1}(s)$. Then the $D^{*}$ set is updated through the evaluation of all the neighbors solutions $s^{\prime \prime} \in N_{1}\left(s^{\prime}\right)$.

\subsubsection{MOVNS algorithm}

The Multi-objective Variable Neighborhood Search (MOVNS) is an algorithm of multi-objective optimization presented by Geiger [18]. Its structure is based on metaheuristic Variable Neighborhood Search
(VNS), delineated by Mladenovic and Hansen [29]. In Algorithm 2 the proposed version of MOVNS, based on Ottoni et al. [24], is presented.

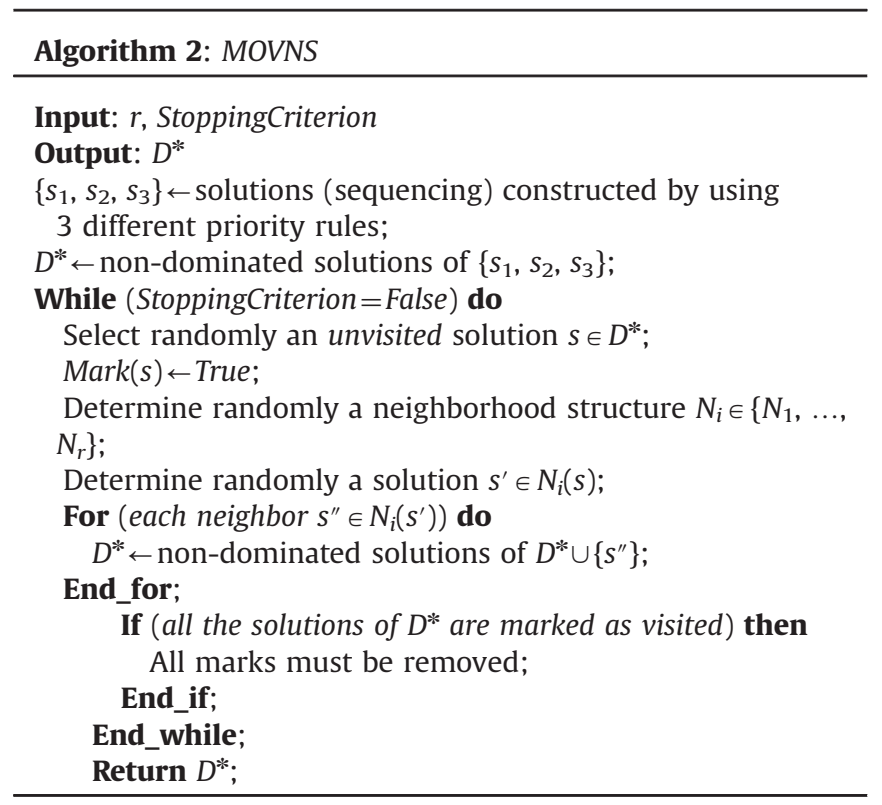

Algorithm 2 starts with the generation of three solutions $\left(s_{1}, s_{2}\right.$ $s_{3}$ ) using the S-SGS described in Section 5.2. Each of these was attained using a different priority rule. These solutions are, then, inter-assessed and, the non-dominated ones are stored in the $D^{*}$ set. Accordingly with Geiger [18], from each local search iteration a non-visited solution $s \in D^{*}$ is randomly selected and marked as visited $\left(\operatorname{Mark}(s) \leftarrow\right.$ True). A neighborhood structure $N_{i} \in\left\{N_{1}, \ldots, N_{r}\right\}$ is also randomly selected. Two neighborhood structures $(r=2)$ were used in Algorithm 2, as described in Section 5.3. After that, a solution $s^{\prime} \in N_{i}(s)$ is randomly determined and the set $D^{*}$ is updated through the assessment of all neighbors solutions $s^{\prime \prime} \in N_{i}\left(s^{\prime}\right)$. Finally, it is checked whether all solutions belonging to $D^{*}$ are marked as visited. If they are, the marking is removed from all solutions. This procedure is repeated until the stopping criterion is fulfilled.

\subsubsection{GMOVNS algorithm}

The GMOVNS proposed in this study is a hybrid algorithm that combines MOG features with MOVNS features, described in Sections 5.4.1 and 5.4.2, respectively. The algorithm follows the structure described in Algorithm 1, but has modifications on the construction and on local search phases. The pseudo-code of GMOVNS is presented in Algorithm 3.

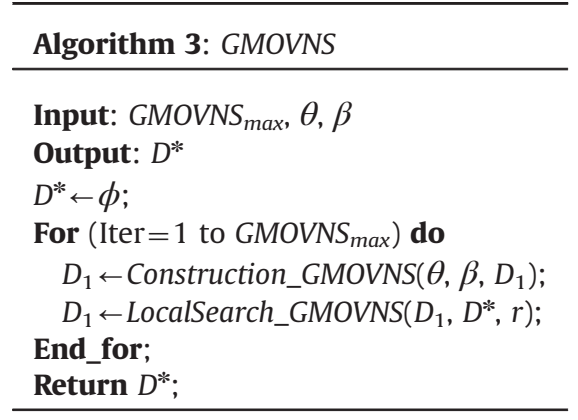

As it is observed in Algorithm 3, the GMOVNS - just like the MOG is composed of two phases: construction and local search. Algorithm 
3.1 describes the Construction_GMOVNS procedure, in which a set of non-dominated solutions $D_{1}$ is generated on each algorithm iteration.

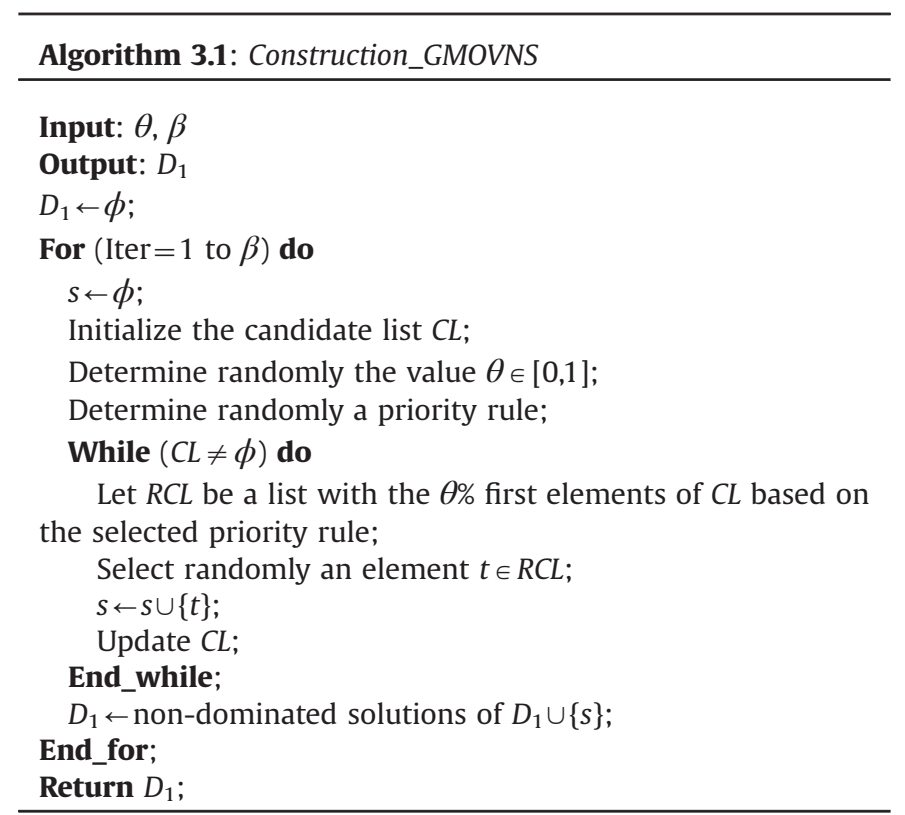

In each one of the GMOVNS $\max$ iterations of Algorithm 3, $\beta$ solutions are generated during the construction phase described in Algorithm 3.1. These solutions are assessed and the nondominated ones are stored in the $D_{1}$ set. All the solutions of this phase are generated through the same adaptation of S-SGS used in $M O G$. For the different solutions to be generated, a value for $\theta \in[0,1]$ and a priority rule are randomly determined during the construction of each solution.

In the local search phase of the GMOVNS, the metaheuristic VNS was proposed with two neighborhood structures, described in Section 5.3 and used in Algorithm 2. The VNS is better capable of exploring the space of feasible solutions to this problem due to its systematic swap of the neighborhood structure. With this, the quality of set $D *$ can be improved. The pseudo-code of the procedure LocalSearch_GMOVNS is presented in Algorithm 3.2.

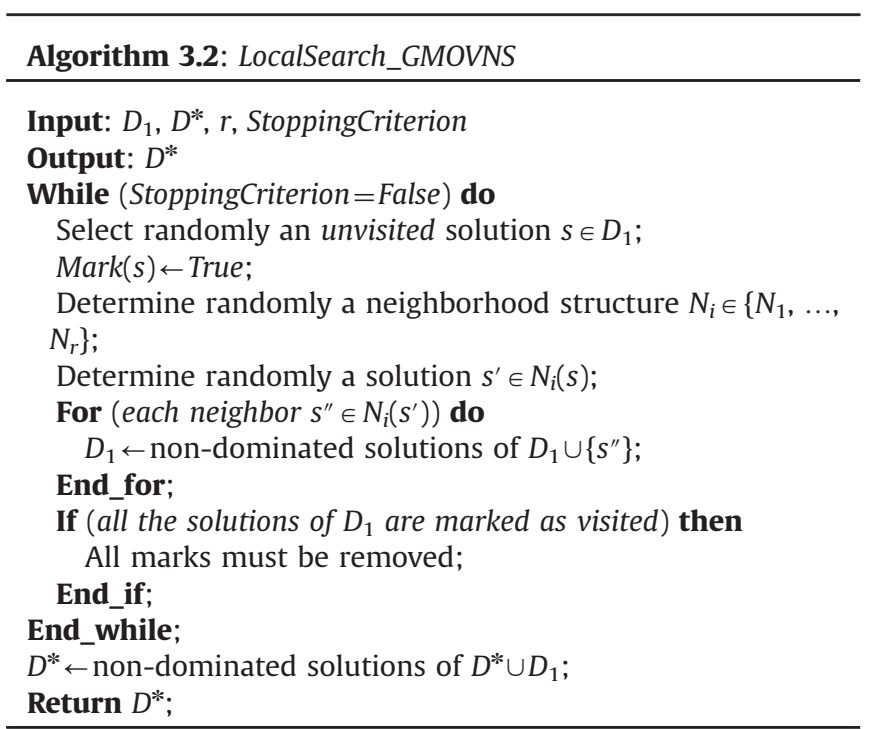

On each iteration of Algorithm 3.2 the solution $s$ to be explored is determined randomly within the non-visited ones that belong to set $D_{1}$ generated in the construction phase. Then, a neighborhood structure $N_{i} \in\left\{N_{1}, \ldots, N_{r}\right\}$ and a neighbor solution $s^{\prime} \in N_{i}(s)$ are chosen randomly. The $D_{1}$ set is then updated through the assessment of all the neighbors solutions $s^{\prime \prime} \in N_{i}\left(s^{\prime}\right)$. Finally, it is checked, if all the solutions that belong to $D_{1}$ are marked as visited, and, if they are, the marking is removed from all solutions. This procedure is repeated until the stopping criterion is fulfilled. From $D_{1}$ on each iteration the $D^{*}$ set is updated with the assessment of all solutions of $D^{*} \cup D_{1}$.

\subsubsection{MOVNS_I algorithm}

Two variants of algorithm MOVNS are found in literature. One is proposed by Ottoni et al. [24] and another by Arroyo et al. [23]. These variants consist of adding an intensification procedure to the algorithm. The intensification of the search around the best solution is obtained, for example by the application of small perturbations on it. The MOVNS with intensification, denominated MOVNS_I, proposed in this work is based on the variant proposed by Ottoni et al. [24] and it is described in Algorithm 4.

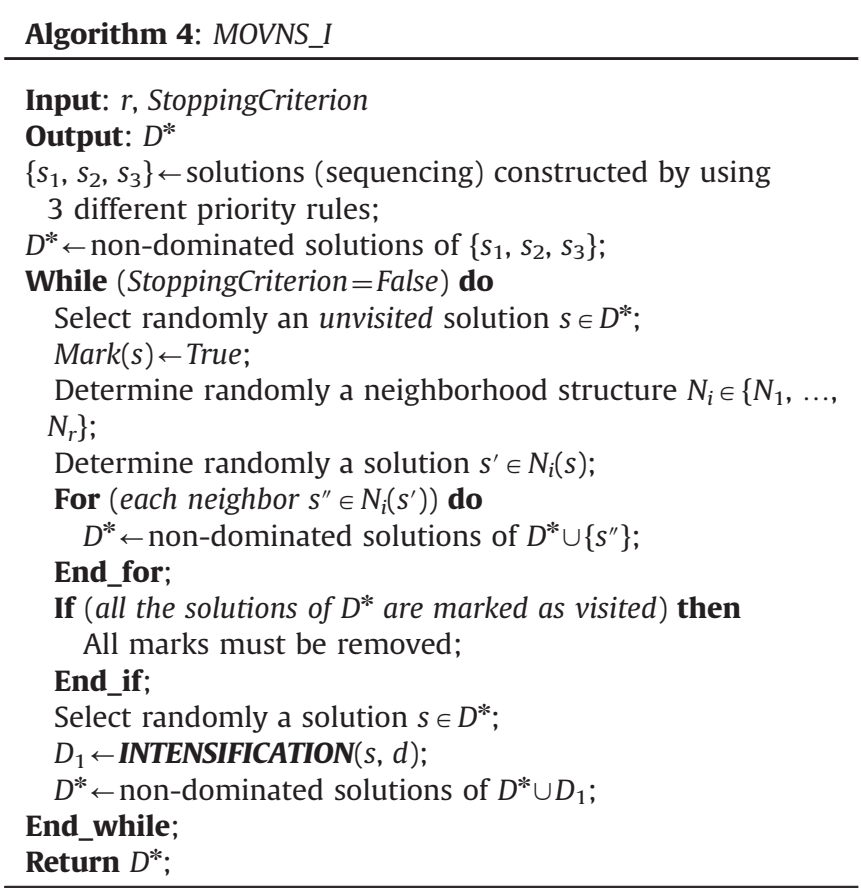

According to Ottoni et al. [24], the intensification procedure is composed by two stages: destruction and reconstruction, as presented in Algorithm 4.1.

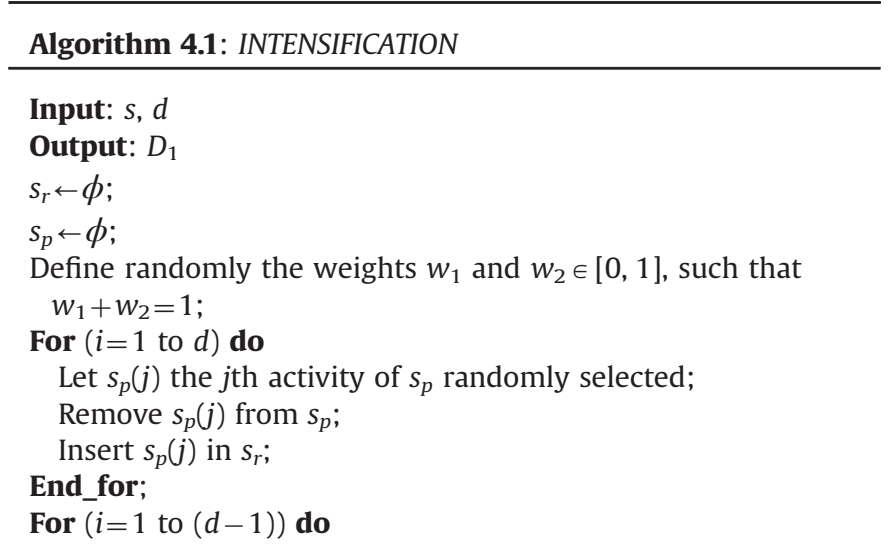




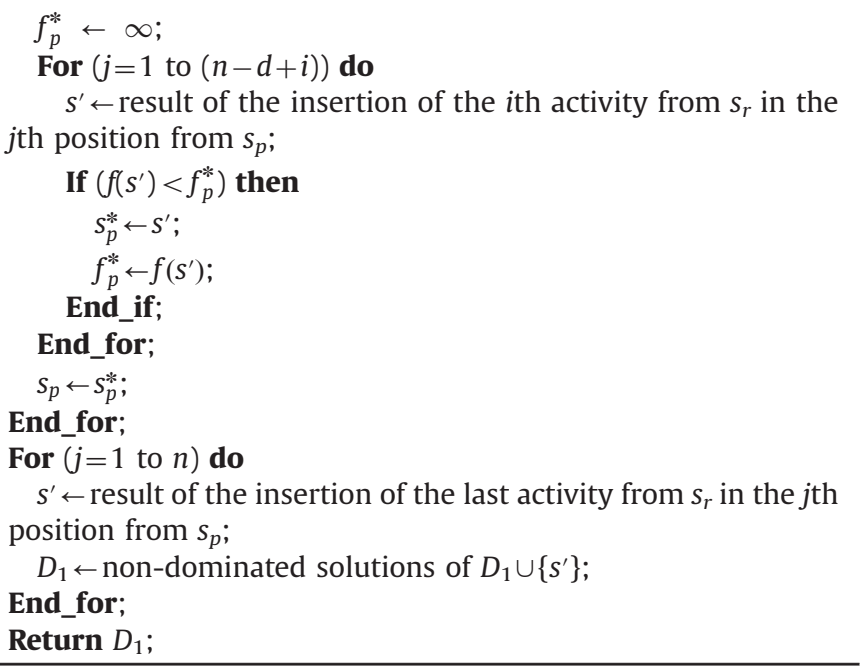

The intensification procedure starts with the destruction stage, in which $d$ activities are removed from a solution $s \in D^{*}$ randomly selected. In our experiments, $d$ was fixed at 4 . This strategy results in the generation of a partial solution $s_{p}$, composed by $(n-d)$ activities, and of a set $s_{r}$ with the $d$ activities removed from $s$. Then the solution $s$ is reconstructed inserting $(d-1)$ activities of $s_{r}$ in $s_{p}$. To do this, an activity belonging to $s_{r}$ is inserted in all possible positions of $s_{p}$. The position that offers the best partial solution is selected. The assessment of the partial solutions is done through a weighted function given by the equation $f=w_{1} f_{1}+w_{2} f_{2}$, where $w_{1}$ and $w_{2}$ are associated weights with the objective functions and $w_{1}+w_{2}=1$. This procedure is made until $(d-1)$ activities of $s_{r}$ are inserted in $s_{p}$. Finally, the last activity of $s_{r}$ is inserted in the partial solution $s_{p}$ in all its possible positions. All solutions generated by this last insertion process are assessed and the non-dominated ones are stored in $D_{1}$.

After the intensification procedure, the set $D *$ is updated through the assessment of all $D^{*} \cup D_{1}$ solutions.

\subsubsection{PILS algorithm}

The Pareto Iterated Local Search (PILS) is a multi-objective optimization algorithm proposed by Geiger [19]. It is based on metaheuristic Iterated Local Search (ILS) delineated by Lourenço et al. [30]. The basic pseudo-code of PILS is presented in Algorithm 5.

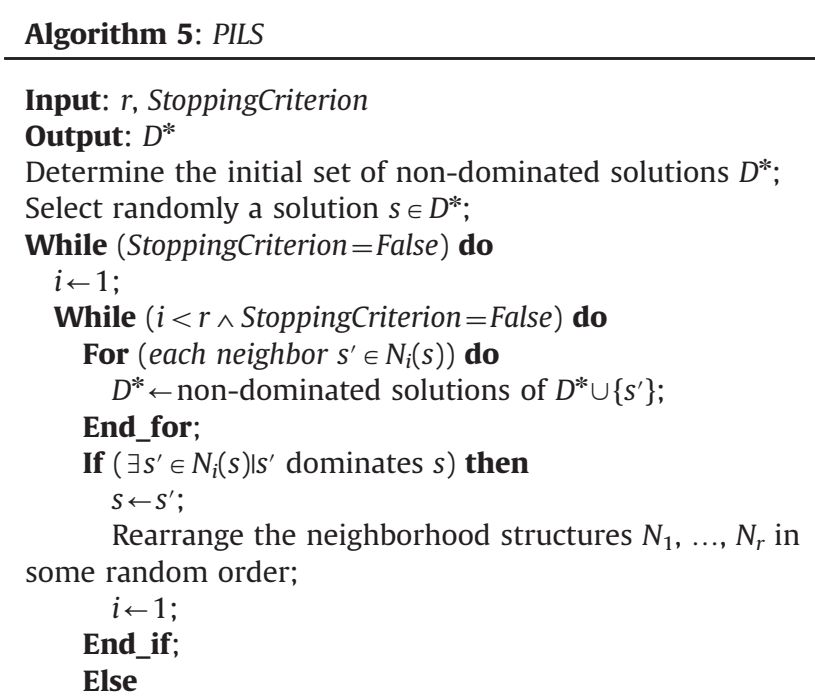

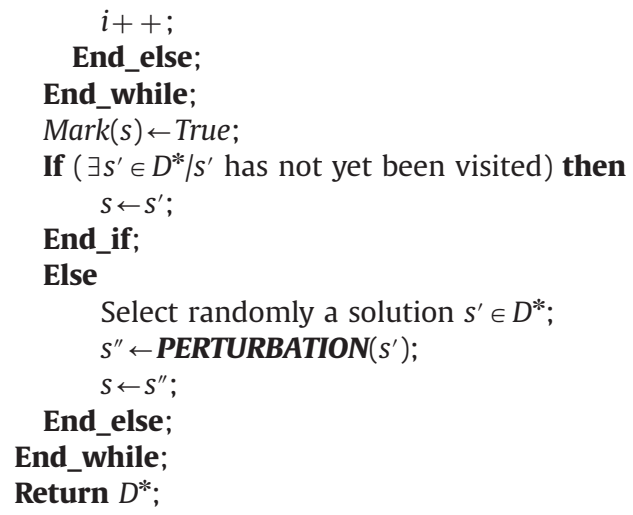

Algorithm 5 starts with the generation of an initial set of nondominated solutions $D^{*}$, using the procedure S-SGS and the priority rules from Section 5.2. After that, a solution $s \in D^{*}$ is randomly selected, that starts to be the current solution and all its neighborhood is explored. The neighborhood structures used are presented on Section $5.3(r=2)$. In case any neighbor solution $s^{\prime}$ $\in N_{i}(s)$ dominates the current solution $s$, then $s^{\prime}$ starts to be the new current solution, the neighborhood structures are then randomly reordered and the procedure returns to its first neighborhood structure of the new generated order. This procedure is repeated until all solutions belonging to $D^{*}$ are visited, that is, until the algorithm arrives in a local optimum in the explored neighborhood. Once this is done, a solution $s^{\prime} \in D^{*}$ is randomly selected on which a perturbation is applied. The objective on perturbation a solution is to explore other local optimums. The perturbation used here is proposed originally by Geiger [19] and works as follows: after the selection of solution $s^{\prime} \in D^{*}$, one position $j \leq n-4$ is randomly determined along with four consecutive activities of $s^{\prime}$ on the positions $j, j+1, j+2$ and $j+3$. A solution $s^{\prime \prime}$ is then generated by applying the activities swap movement on positions $j$ and $j+3$, and on the activities from positions $j+1$ and $j+2$. Thus, the activities before the activity on position $j$ and those before the activity on position $j+3$, stay on the same position after the perturbation. After that the solution $s^{\prime \prime}$ starts to be the current solution and its neighborhood is explored. In case all neighbors solutions from the one generated by the perturbation are dominated by any solution that belongs to $D^{*}$, then the perturbation procedure is repeated. This procedure is repeated until the stopping criterion is fulfilled.

\section{Computational experiments}

The five algorithms presented in this study were coded in $\mathrm{C}++$ and executed on an AMD Turion II Dual-Core with a $2.20 \mathrm{GHz}$ and 4.0 GB of RAM.

The algorithms were run with the same stopping criterion (StoppingCriterion) based on the limit of the generated solutions. In literature, this stopping criterion is extensively used for performance comparison of mono and multi-objective algorithms for the RCPSPRP, as illustrated in [31,6, 32] and others. Several values are found in literature, but in this work the limit of generated solutions equal to 5000 was used as the stopping criterion for the algorithms.

In the execution of the MOG algorithm the value 100 for the $M O G_{\max }$ parameter was empirically defined. For the execution of GMOVNS, the value 10 to $\beta$ and the value 100 to the GMOVNS $\max$ also were empirically defined. 


\subsection{Problems instances}

According to Viana and Sousa [12] the study of multi-objective RCPSPRP involves some difficulties, specially related to the availability of instances shown in literature. Several mono-objective problems can be found, like the Project Scheduling Problem Library (PSPLib), developed by Kolisch and Sprecher [33], but nothing was found by the authors regarding multi-objective instances.

Due to this, 160 instances from the PSPLib, available in [34], were used to test the algorithms. These instances have the numbers of activities $n=30,60,90$ and 120. For each value of $n$, 40 instances were used, from which 4 different types of renewable resources are available. As the instances were used for the monoobjective RCPSPRP and they do not present associated weights to the activities. Thus, such weights were then generated randomly and uniformly distributed over the interval $[1,500]$.

Due to the fact the proposed algorithms using random choices, the five algorithms were run thirty times independently (replicates), with thirty different seeds randomly generated, for all the 160 instances. From the solutions attained on the thirty runs of each algorithm, the sets of non-dominated solutions are determined for each instance.

\subsection{Performance measures}

The comparison between non-dominated solution sets attained by multi-objective optimization algorithms is not a trivial task. Several performance measures (metrics) of multi-objective algorithms can be found in literature, such as in [35-38] and [39].

In this work, to assess the quality of the non-dominated solutions attained by the proposed algorithms, four multi-objective performance measures were used: distance metrics, hypervolume indicator, epsilon metric and error ratio.

For each instance $D_{i}$ is the non-dominated solutions set found by the algorithm $i$, for $i=1,2, \ldots, h$, and $h$ is the number of assessed algorithms. From these sets a reference set, denoted by Ref, where $R e f=\left\{s \in D_{1} \cup D_{2} \cup \ldots \cup D_{h} \mid s\right.$ is a non-dominated solution $\}$, is determined. The Ref set is the best known Pareto-optimal front. The performance of an algorithm is then measured in terms of the quality of the solution obtained by this algorithm regarding the solutions in Ref. Based on the Ref set, the definition of the used performance measures are presented as follows:

Distance metrics: measures the proximity between the solutions of set $D_{i}$ and the solutions of set Ref. It also measures the solutions spreading on set $D_{i}$. The closer to zero the distances are, the better the quality of the solutions found by the algorithm will be. The formulas used to calculate the average $\left(D_{a v}\right)$ and maximum $\left(D_{\max }\right)$ distances from the $D_{i}$ solutions compared to the Ref set are:

$$
\begin{aligned}
& D_{a v}\left(D_{i}\right)=100 \times \frac{1}{|\operatorname{Ref}|_{s}} \sum_{\operatorname{Ref}} \min _{s^{\prime} \in D_{i}} d\left(s, s^{\prime}\right) \\
& D_{\max }\left(D_{i}\right)=\max _{s \in \operatorname{Ref}}\left\{\min _{s^{\prime} \in D_{i}} d\left(s, s^{\prime}\right)\right\} \times 100
\end{aligned}
$$

in which $|R e f|$ is the cardinality of set Ref and:

$$
\left(s, s^{\prime}\right)=\max \left\{\frac{\left(f_{1}(s)-f_{1}\left(s^{\prime}\right)\right)}{\Delta_{1}}, \frac{\left(f_{2}(s)-f_{2}\left(s^{\prime}\right)\right)}{\Delta_{2}}\right\}
$$

$\Delta_{j}$ is the difference between the biggest and the smallest value of the objective function $f_{j}$, considering the solutions of set Ref.

The distances $D_{a v}$ and $D_{\max }$ are broadly used as performance measure of multi-objective algorithms such as in $[9,12]$ and [24].
Hypervolume indicator: measures the covered or dominated area by set $D_{i}$. For the minimization of two objectives, a reference point $(x, y)$ is used to limit this coverage, denoted by $H\left(D_{i}\right)$, where $x$ and $y$ are upper bounds for $f_{1}$ and $f_{2}$, respectively. A larger dominance area indicates that the solutions attained by the algorithm generated a good coverage on the Pareto-optimal front. The value of the hypervolume difference $\left(H^{-}\left(D_{i}\right)\right)$ is calculated by Eq. (6):

$H^{-}\left(D_{i}\right)=H(\operatorname{Ref})-H\left(D_{i}\right)$

As $H(\operatorname{Ref})>H\left(D_{i}\right)$, the smaller the value of $H^{-}\left(D_{i}\right)$, the better the quality of set $D_{i}$ will be. In Fig. 2, the covered area by the solution sets $D_{1}$ and $D_{2}$ are illustrated.

As it is shown in Fig. 2, $H\left(D_{1}\right)>H\left(D_{2}\right)$, therefore $H^{-}\left(D_{1}\right)$ $<H^{-}\left(D_{2}\right)$, which indicates the solutions from the $D_{1}$ set are "better" than the ones from the $D_{2}$ set.

Epsilon metric: given a set $D_{i}$ and $z^{a}=\left(z_{1}^{a}, \ldots, z_{r}^{a}\right)$ and $z^{b}=\left(z_{1}^{b}, \ldots, z_{r}^{b}\right)$, two solutions belonging to the sets $D_{i}$ and Ref, respectively, the epsilon metric denoted by $I_{\varepsilon}^{1}=\left(D_{i}\right)$, measures the maximum normalized distance from set $D_{i}$ in relation to set Ref, and is calculated by Eq. (7):

$I_{\varepsilon}^{1}\left(D_{i}\right)=\max _{Z^{b} \in \operatorname{Ref}}\left\{\min _{Z^{a} \in D_{i}}\left\{\max _{1 \leq j \leq r} \frac{Z_{j}^{a}}{Z_{j}^{b}}\right\}\right\}$

Therefore, the quality of a non-dominated solutions set $D_{i}$ attained by an algorithm to a determined instance is assessed in relation to set Ref and as $I_{\varepsilon}^{1}=\left(D_{i}\right)$ measures the maximum distance of $D_{i}$ in relation to Ref, thus a value close to zero of $I_{\varepsilon}^{1}=\left(D_{i}\right)$ indicates a good quality of set $D_{i}$. To use the epsilon metric to assess a $D_{i}$ set, the values of the objective functions must be normalized according to the following equation:

$f_{i}^{*}(s)=\left(\frac{f_{i}(s)-f_{i}^{\min }}{f_{i}^{\max }-f_{i}^{\min }} \times 100\right)$

where $f_{i}^{\min }$ and $f_{i}^{\max }$ are, respectively, the smallest and the biggest value found to the $i$ th objective considering the solutions belonging to set Ref. Hence, the values of the objective function $f_{i}^{*}(s)$ calculated by Eq. $(8)$ are in the interval $[0,100]$. Error ratio: indicates the percentage of the solutions that belong to set $D_{i}$ that do not belong to set Ref. The metric based on Veldhuizen [36] and denoted by $T E_{i}$, is calculated by Eq. (9):

$T E_{i}=\frac{\left|D_{i}\right|-\left|\operatorname{Ref} \cap D_{i}\right|}{\left|D_{i}\right|} \times 100$

where $\left|D_{i}\right|$ corresponds to the cardinality of set $D_{i}$ and $\left|\operatorname{Re} f \cap D_{i}\right|$ to the number of reference solutions originating from the set $D_{i}$. According to Coello and Lamont [40], $T E_{i}=0$ indicates that

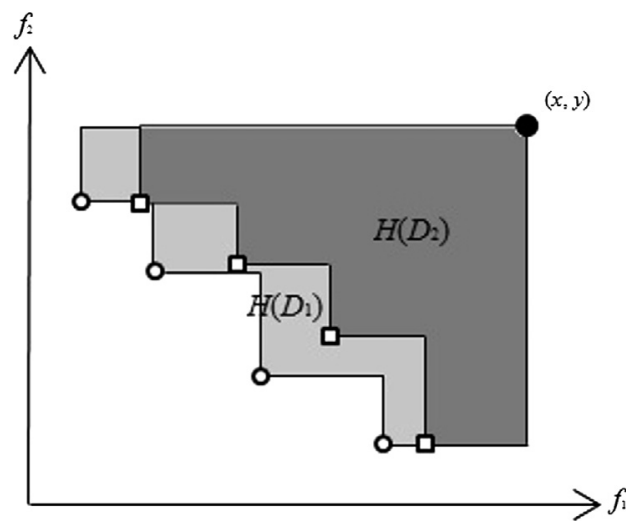

Fig. 2. Examples of areas covered by two sets of solutions. 
all solutions belonging to $D_{i}$ are part of Ref. On the other hand, $T E_{i}=100$ indicates that no solutions from $D_{i}$ are part of Ref. Thus, the nearest to zero the value of the $T E_{i}$ the better is the performance of the algorithm.

\subsection{Computational results}

For each group of 40 instances of size $n$, Table 2 shows the average values (in seconds) of the computational time spent by each algorithm to obtain the non-dominated solutions sets.

Table 2 shows that all algorithms presented low computational effort, i.e., obtained the sets of non-dominated solutions in an acceptable time.

Except Table 5, all following tables in this section presents, for each group of 40 instances of size $n$, the average values of the performance measure attained by each algorithm.

In Tables 3 and 4 the results attained by the algorithms in relation to the distance metrics are presented. In Table 3 the results regarding the average distance and in Table 4 the results regarding the maximum distance are presented.

Through Tables 3 and 4 , it is verified that the MOVNS_I algorithm is the one that produces lower average values, that is, closer to zero, from the average and maximum distances to the majority set of instances. The MOVNS_I did not attain lower average values to the set of instances with $n=60$ only where PILS showed better results.

As presented in Section 6.2, the distance metrics measures the proximity between the solutions of a set $D_{i}$ and the solutions of set Ref. Therefore, the higher the percentage of solutions of $D_{i}$ in the Ref set, the lower tends to be the values of the distance metrics. The values of the distance metrics tend to be smaller, but those values also depend of the distance between $D_{i}$ solutions and solutions belonging to Ref set obtained by other algorithms. For each group of 40 instances of size $n$, Table 5 shows average percentages of solutions obtained by the MOVNS_I and PILS algorithms which are part of Ref set.

Table 5 shows that algorithms had presented very close values for the average percentage except for the set with $n=60$. In this case, the percentage difference was $28.42 \%$. For the groups of instances in which the difference between the average percentages was small, the MOVNS_I algorithm had presented better results for $D_{a v}$ and $D_{\max }$, even the PILS showing higher percentage. However, when the difference between these average percentages was large, as in the case of the instances set with $n=60$, better values for the distances was obtained by the PILS. Therefore, the MOVNS_I had presented in most cases a better performance regarding the distance metrics.

In Table 6 the values attained by the proposed algorithms regarding the hypervolume indicator are presented.

Through Table 6 it is verified that the GMOVNS algorithm presented lower average values, compared with the other algorithms, from the hypervolume indicator for all sets of instances.

In Table 7 the results attained by the proposed algorithms are shown regarding the epsilon metric.

Table 2

Average computational time.

\begin{tabular}{rllllr}
\hline $\boldsymbol{n} n$ & \multicolumn{6}{l}{ Algorithm } & & & \\
\cline { 2 - 6 } & MOG & MOVNS & GMOVNS & MOVNS_I & \multicolumn{1}{c}{ PILS } \\
\hline 30 & 0.18 & 0.42 & 0.39 & 1.05 & 1.12 \\
60 & 1.01 & 3.93 & 3.01 & 4.12 & 10.98 \\
90 & 2.78 & 13.21 & 11.04 & 15.79 & 53.44 \\
120 & 7.69 & 54.13 & 36.40 & 57.70 & 143.34 \\
\hline
\end{tabular}

Table 3

Distance metrics results - average distance (\%).

\begin{tabular}{lllllr}
\hline $\boldsymbol{n}$ & \multicolumn{2}{l}{ Algorithm } & & \\
\cline { 2 - 6 } & MOG & MOVNS & GMOVNS & MOVNS_I & PILS \\
\hline 30 & 15.92 & 18.30 & 13.86 & 3.71 & 6.17 \\
60 & 64.84 & 19.17 & 19.12 & 13.64 & 5.80 \\
90 & 14.55 & 14.55 & 15.11 & 6.50 & 9.87 \\
120 & 32.67 & 16.86 & 26.50 & 3.41 & 12.64 \\
Average & 31.99 & 17.44 & 18.65 & 6.81 & 8.62 \\
\hline
\end{tabular}

Table 4

Distance metrics results - maximum distance (\%).

\begin{tabular}{llllll}
\hline $\boldsymbol{n}$ & \multicolumn{2}{l}{ Algorithm } & & & \\
\cline { 2 - 6 } & MOG & MOVNS & GMOVNS & MOVNS_I & PILS \\
\hline 30 & 64.75 & 49.10 & 55.80 & 12.50 & 20.25 \\
60 & 102.49 & 62.82 & 78.55 & 35.51 & 21.00 \\
90 & 47.55 & 40.64 & 38.34 & 14.80 & 29.90 \\
120 & 88.27 & 44.40 & 95.93 & 14.15 & 37.23 \\
Average & 75.76 & 49.24 & 67.15 & 19.24 & 27.01 \\
\hline
\end{tabular}

Table 5

Average Percentages of Solutions of the MOVNS_I and PILS in the Ref Set.

\begin{tabular}{rlrr}
\hline $\boldsymbol{n}$ & \multicolumn{2}{l}{ Algorithm } & Difference \\
\cline { 2 - 3 } & MOVNS_I & PILS & \\
\hline 30 & 56.70 & 59.80 & 3.10 \\
60 & 27.10 & 55.52 & 28.42 \\
90 & 36.67 & 38.63 & 1.96 \\
120 & 42.12 & 44.22 & 2.10 \\
\hline
\end{tabular}

Table 6

Hypervolume indicator results.

\begin{tabular}{lrrrrr}
\hline $\boldsymbol{N}$ & \multicolumn{2}{l}{ Algorithm } & & & \\
\cline { 2 - 6 } & \multicolumn{1}{l}{ MOG } & MOVNS & GMOVNS & \multicolumn{1}{c}{ MOVNS_I } & \multicolumn{1}{c}{ PILS } \\
\hline 30 & 925.78 & 616.44 & 352.12 & 373.22 & 367.21 \\
60 & 1769.16 & 807.31 & 535.98 & 1433.66 & 888.32 \\
90 & 2700.65 & 2351.28 & 1979.39 & 2143.45 & 2132.65 \\
120 & 5165.89 & 5913.00 & 3715.94 & 3876.98 & 3800.99 \\
Average & 2640.37 & 2422.01 & 1645.86 & 1956.83 & 1797.29 \\
\hline
\end{tabular}

Through Table 7 it is verified that the GMOVNS is the algorithm that produces lower average values for the epsilon metric for all sets of instances.

In Table 8, the values attained by the algorithms proposed regarding the error ratio are presented.

As it can be observed in Table 8, the PILS algorithms presented, in all sets of instances, a lower average value for the error ratio. This means that, based on error ratio, the algorithm PILS was superior to the others.

\subsubsection{Analysis of the results}

Based on the average values of the computational time spent by each algorithm to obtain the non-dominated solutions sets, we can see that all the algorithms were computationally efficient, obtaining solutions sets in an acceptable time. For all the instances sets, 
Table 7

Epsilon metric results.

\begin{tabular}{llllll}
\hline $\boldsymbol{N}$ & \multicolumn{2}{l}{ Algorithm } & & & \\
\cline { 2 - 6 } & MOG & MOVNS & GMOVNS & MOVNS_I & PILS \\
\hline 30 & 1.47 & 1.93 & 1.21 & 1.82 & 1.25 \\
60 & 1.44 & 1.75 & 1.28 & 1.50 & 1.44 \\
90 & 1.88 & 1.85 & 1.58 & 1.91 & 1.80 \\
120 & 1.36 & 1.70 & 1.21 & 1.90 & 1.50 \\
Average & 1.54 & 1.81 & 1.30 & 1.78 & 1.50 \\
\hline
\end{tabular}

Table 8

Error ratio results (\%).

\begin{tabular}{llllll}
\hline $\boldsymbol{n}$ & \multicolumn{2}{l}{ Algorithm } & & \\
\cline { 2 - 6 } & MOG & MOVNS & GMOVNS & MOVNS_I & PILS \\
\hline 30 & 77.20 & 40.72 & 59.62 & 43.30 & 40.20 \\
60 & 86.19 & 74.65 & 66.94 & 72.90 & 44.48 \\
90 & 89.65 & 62.11 & 75.15 & 63.33 & 61.37 \\
120 & 91.47 & 90.52 & 67.01 & 57.88 & 55.78 \\
Average & 86.13 & 67.00 & 67.18 & 59.35 & 50.46 \\
\hline
\end{tabular}

the MOG and PILS algorithms had presented the lowest and highest average computational time, respectively.

Results attained from the computational experiments, showed that the GMOVNS algorithm had best performance. The GMOVNS has generated better results for two of the four multi-objective performance measures assessed: hypervolume indicator and epsilon metric. This means that the GMOVNS algorithm produces a better coverage for the Pareto-optimal front and that the nondominated solutions generated by this algorithm are closer to the Ref set. Regarding the distance metrics, in general, the MOVNS_I algorithm has obtained the lowest average values for these metrics. Therefore, the MOVNS_I has achieved better distributed solutions throughout the Ref set. For all the instances sets, the PILS algorithm had obtained the better results for the error ratio. The PILS had presented, on average, the higher percentage of solutions belonging to the Ref set.

Table 9 shows a comparison between the results obtained by the proposed algorithms and those obtained by Viana and Sousa [12]. This comparison was made regarding the average values obtained for the distance metrics, for instances with 30 activities from PSPLib.

Through Table 9 it is verified that all the proposed algorithms presented lowest computational effort compared with the proposed algorithms by Viana and Sousa [12]. Regarding the distance metrics, the MOVNS_I and PILS have obtained better results, that is, values closer to zero, compared to the PSA and MOTS proposed by Viana e Sousa [12]. Therefore, the MOVNS_I and PILS have achieved better distributed solutions throughout the Ref set. Only the MOVNS has obtained worst results for the two distances, compared with the MOTS.

Table 10 presents a comparison between the results obtained by the proposed algorithms and those obtained by Ballestín and Blanco [6]. The comparison was performed using the average values obtained for the average distance metric, for instances with 120 activities from PSPLib. The used results were obtained by Ballestín and Blanco [6] limiting the number of generated solutions in 5000, the same way that in this work.

Through Table 10 it is verified that, except the MOG, all the other proposed algorithms have obtained better results for the average distance, that is, values closer to zero, compared to the SPEA-II,
Table 9

Comparison of average values for $D_{a v}, D_{m z x}$ and computational time (in seconds).

\begin{tabular}{lccc}
\hline Algorithm & $\boldsymbol{D}_{\boldsymbol{a v}}$ & $\boldsymbol{D}_{\boldsymbol{m a x}}$ & Computational time \\
\hline MOG & 15.92 & 64.75 & 0.18 \\
MOVNS & 18.30 & 49.10 & 0.42 \\
GMOVNS & 13.86 & 55.80 & 0.39 \\
MOVNS_I & 3.71 & 12.50 & 1.05 \\
PILS & 6.17 & 20.25 & 1.12 \\
PSA [12] & 32.19 & 41.76 & 2590 \\
MOTS [12] & 16.63 & 33.86 & 2599 \\
\hline
\end{tabular}

Table 10

Comparison of average values for $D_{a v}$.

\begin{tabular}{lr}
\hline Algorithm & $\boldsymbol{D}_{\boldsymbol{a} \boldsymbol{v}}$ \\
\hline MOG & 32.67 \\
MOVNS & 16.86 \\
GMOVNS & 26.50 \\
MOVNS_I & 3.41 \\
PILS & 12.64 \\
SPEA-II [6] & 27.00 \\
NSGA-II [6] & 27.00 \\
PSA [6] & 53.00 \\
\hline
\end{tabular}

NSGA-II and PSA proposed by Ballestín and Blanco [6]. The MOG has obtained better results only compared to the PSA. Therefore, the MOVNS, GMOVNS, MOVNS_I and PILS have achieved better distributed solutions throughout the Ref set.

To compare results, no work was found in literature using the hypervolume indicator, epsilon or error ratio to assess multiobjective algorithms applied to the RCPSPRP.

\subsection{Statistical analysis}

The experiments that follow aim at verifying, if there is a significant difference between the algorithms proposed in this paper, concerning the multi-objective performance measures used. These experiments were conducted with the assistance of the Minitab $®$ computational package on its 16th version. It is emphasized here that this experimentation enables the researchers to make inferences to the population of all instances.

To conduct the experiments, the statistical technique Analysis of Variance (ANOVA) was chosen, as described by Montgomery [41]. The interest is then to test the equality of the population means $(\mu)$ to the five implemented algorithms against the inequality of the means.

In the ANOVA application two hypotheses were tested:

$H_{0}: \mu_{1}=\mu_{2}=\mu_{3}=\mu_{4}=\mu_{5}$

$H_{1}: \mu_{i} \neq \mu_{j}$, for at least one pair $(i, j)$, with $i, j=1,2,3,4,5$ and $i \neq j$

In tests (10) and (11), the null hypothesis (10) represents the equality of the population means hypothesis in relation to the analyzed multi-objective performance measure on the five algorithms, that is, it conjectures that there is no significant difference between these algorithms regarding the metric. Hypothesis (11), on the other hand, conjectures the opposite.

However, to apply the ANOVA, the sample data should be normally distributed in this case, and the population variances $\left(\sigma^{2}\right)$ approximately equal between the factor levels, regarding the algorithms proposed here.

Although the test is based on the supposition that the sample data should be normally distributed, according to Kulinskaya et al. [42], 
this hypothesis is not critical when the sizes of the samples are at least 15 or 20. Once all the samples on this work have the equal size to 160 (number of instances used) for each algorithm, thus, the normality is not critical. Hence, the normality premise is verified for all the algorithms regarding all metrics. To use the ANOVA it is needed, then, the verification of only the variances proximity between the data from the algorithms regarding each metrics. For this, the following hypotheses were tested:

$H_{0}: \sigma_{1}^{2}=\sigma_{2}^{2}=\sigma_{3}^{2}=\sigma_{4}^{2}=\sigma_{5}^{2}$

$H_{1}: \sigma_{i}^{2} \neq \sigma_{j}^{2}$ for at least one pair $(i, j)$, with $i, j=1,2,3,4,5$ and $i \neq j$

In tests (12) and (13), the null hypothesis (12) represents the equality of the population variances hypothesis in relation to the analyzed multi-objective performance measure on the five algorithms. Hypothesis (13) conjectures the opposite.

By applying these hypothesis tests is possible to calculate a test statistic that allows us to accept or reject the null hypothesis. In Statistical Inference is usual to represent this test statistic for $p$-value. From the value of this test statistic and of a criterion for acceptance/rejection is possible to conclude, with a significance level $\alpha$ defined a priori, which of the hypotheses accept. That is, if $\alpha \geq p$-value rejects $H_{0}$. All the tests in this section have been executed with a significance level $\alpha=0.05$ (5\%).

Nevertheless, the ANOVA does not tell us which pairs of algorithms present significant differences, that is, result in different means to each assessed metric. To answer this question the method of the Least Significant Difference (LSD), also known as Fisher's method [41], is used.

All the tables of ANOVA results presented in this section show the calculated value of the $p$-value, the sample means, the sample standard deviations and the interval limits with $95 \%$ of confidence on the population means from the analyzed multi-objective performance measure, in accordance with each algorithm.

\section{- Distance Metrics}

For the distance metrics the hypothesis tests (12) and (13) were used to verify the proximity of variances between the data of all algorithms. The $p$-value statistics calculated for this test was equal to 0.075 for the average distance, and 0.055 for the maximum distance. Once $\alpha<p$-value for both distance metrics, the variance equality hypothesis is accepted between the population data to the five algorithms. Therefore, once the premise is verified, the ANOVA is applied to the concerning data from the metrics.

The application of ANOVA to the average distance data allowed us to calculate the values presented in Table 11 .

According to the results in Table $11, p$-value $=0.027$. Therefore, it can be stated that the null hypothesis should be rejected, that is, as $\alpha \geq p$-value, there are enough statistical evidences to conclude that the average values regarding the average distance are different on each algorithm. By using the LSD method, it can be stated that there are statistical evidences showing that the average values, regarding the average distance, are different within the following algorithm pairs: $M O G \times$ MOVNS_I and MOG $\times$ PILS.

The application of ANOVA to the maximum distance data allowed us to calculate the values presented in Table 12 .

According to the results in Table $12, p$-value $=0.004$. Therefore, it can be stated that the null hypothesis should be rejected, that is, as $\alpha \geq p$-value, there are enough statistical evidences to conclude that the average values regarding the maximum distance are different on each algorithm. By using the LSD method, it can be stated that there are statistical evidences showing that the average values regarding the maximum distance, are different within the following algorithm pairs: $M O G \times M O V N S, M O G \times$ MOVNS_I and MOG $\times$ PILS.

- Hypervolume Indicator

For the hypervolume indicator it was verified the proximity of variances between the data of all algorithms by the hypothesis tests (12) and (13). The calculated $p$-value statistics was equal to 0.560 and, as $\alpha<p$-value, the hypothesis of the variances equality between the population data on the five algorithms is accepted. Once verified the premise, the ANOVA is applied to the data of this metric.

The application of ANOVA to the hypervolume indicator data allowed us to calculate the values presented in Table 13 . According to the results in Table $13, p$-value $=0.443$. Therefore, it can be stated that the null hypothesis should be accepted, that is, as $\alpha<p$-value, there are enough statistical evidences to conclude, with a 5\% significance level ( $\alpha=0.05)$, that the average values regarding the hypervolume indicator equal within all algorithms.

- Epsilon metric

For the epsilon metric it was verified the proximity of variances between the data of all algorithms by the hypothesis tests (12) and (13). The calculated $p$-value statistics was equal to 0.077 and, as $\alpha<p$-value, the hypothesis of the variances equality between the population data on the five algorithms is accepted. Once verified the premise, the ANOVA is applied to the data of this metric.

The application of ANOVA to the epsilon metric data allowed us to calculate the values presented in Table 14

According to the results in Table 14, $p$-value $=0.026$. Therefore, it can be stated that the null hypothesis should be rejected, that is, as $\alpha \geq p$-value, there are enough statistical evidences to conclude that the average values regarding the epsilon metric are different between the algorithms. By using the LSD method, it can be stated that there are statistical evidences showing that the average values, regarding the epsilon metric, are different within the following algorithm pairs: GMOVNS $\times$ MOVNS and GMOVNS $\times$ MOVNS_I.

- Error ratio

For the error ratio the hypothesis tests (12) and (13) were used to verify the proximity of variances between the data of all algorithms. The calculated $p$-value statistics was equal to 0.008 and, as $\alpha>p$-value, the hypothesis of the variances equality between the population data on the five algorithms is rejected. Therefore, this premise is not verified, and consequently, the

Table 11

The results of ANOVA for the average distance.

\begin{tabular}{|c|c|c|c|c|c|}
\hline \multirow{2}{*}{$\begin{array}{l}\text { p-Value, } \\
0.027\end{array}$} & \multicolumn{5}{|l|}{ Algorithm } \\
\hline & $M O G$ & MOVNS & GMOVNS & MOVNS_I & PILS \\
\hline Mean & 32.0 & 17.4 & 18.6 & 6.8 & 8.6 \\
\hline Standard deviation & 102.8 & 29.3 & 31.5 & 10.5 & 10.8 \\
\hline IC $(\mu, 95 \%)$ & $(5.7 ; 72.0)$ & $(8.0 ; 26.1)$ & $(8.3 ; 29.0)$ & $(3.7 ; 9.9)$ & $(5.1 ; 12.3)$ \\
\hline
\end{tabular}


Table 12

The results of ANOVA for the maximum distance.

\begin{tabular}{|c|c|c|c|c|c|}
\hline \multirow{2}{*}{$\begin{array}{l}\text { p-Value, } \\
0.004\end{array}$} & \multicolumn{5}{|l|}{ Algorithm } \\
\hline & MOG & MOVNS & GMOVNS & MOVNS_I & PILS \\
\hline Mean & 75.8 & 49.2 & 67.1 & 19.2 & 27.0 \\
\hline Standard deviation & 161.6 & 59.2 & 129.8 & 19.7 & 27.9 \\
\hline IC $(\mu, 95 \%)$ & $(19.9 ; 143.1)$ & $(29.8 ; 68.1)$ & $(24.9 ; 106.6)$ & $(12.5 ; 25.6)$ & $(18.0 ; 35.9)$ \\
\hline
\end{tabular}

Table 13

The results of ANOVA for the hypervolume indicator.

\begin{tabular}{|c|c|c|c|c|c|}
\hline \multirow{2}{*}{$\begin{array}{l}\text { p-Value, } \\
0.443\end{array}$} & \multicolumn{5}{|l|}{ Algorithm } \\
\hline & $M O G$ & MOVNS & GMOVNS & MOVNS_I & PILS \\
\hline Mean & 2640.4 & 2422.0 & 1645.9 & 1956.8 & 1797.3 \\
\hline Standard deviation & 2991.5 & 3032.4 & 2275.5 & 2768.2 & 2788.9 \\
\hline IC $(\mu, 95 \%)$ & $(1645.3 ; 3654.9)$ & $(1290.7 ; 3296.3)$ & $(934.3 ; 2416.2)$ & $(1105.3 ; 2812.2)$ & $(865.2 ; 2694.1)$ \\
\hline
\end{tabular}

Table 14

The results of ANOVA for the Epsilon metric.

\begin{tabular}{|c|c|c|c|c|c|}
\hline \multirow{2}{*}{$\begin{array}{l}\text { p-Value, } \\
0.026\end{array}$} & \multicolumn{5}{|l|}{ Algorithm } \\
\hline & $M O G$ & MOVNS & GMOVNS & MOVNS_I & PILS \\
\hline Mean & 1.54 & 1.81 & 1.30 & 1.78 & 1.50 \\
\hline Standard deviation & 0.82 & 0.95 & 0.47 & 0.82 & 0.56 \\
\hline IC $(\mu, 95 \%)$ & $(1.29 ; 1.80)$ & $(1.45 ; 2.10)$ & $(1.15 ; 1.45)$ & $(1.50 ; 2.06)$ & $(1.32 ; 1.67)$ \\
\hline
\end{tabular}

ANOVA cannot be applied to this metric's data. As a result, the Kruskal-Wallis non-parametric test [43] was used. The difference from ANOVA to the Kruskal-Wallis non-parametric test is that the later, instead of working with means, uses population medians $(\eta)$. The test can be used to verify the medians equality of two or more populations and, applying to this work, tests the following hypothesis:

$H_{0}: \eta_{1}=\eta_{2}=\eta_{3}=\eta_{4}=\eta_{5}$

$H_{1}: \eta_{i} \neq \eta_{j}$ for at least one pair $(i, j)$, with $i$,

$j=1,2,3,4,5$ and $i \neq j$

In tests (14) and (15), the null hypothesis (14) represents the equality of the population medians hypothesis in relation to the error ratio on the five algorithms, that is, it conjectures that there is no significant difference between these algorithms regarding this metric. Hypothesis (15), on the other hand, conjectures the opposite.

For this test of hypothesis, the $p$-value statistics calculation brought the value 0.001 . Once the significance level $\alpha=0.05$ is adopted and $\alpha>p$-value, the median equality between the population data on the five algorithms should be rejected. Hence, it is statistically concluded that the algorithms differ in error ratio. By comparing the pairs of algorithms, it can be stated that there are statistical evidences that the median values from the error ratio are different between: MOG $\times$ MOVNS, $M O G \times G M O V N S, M O G \times M O V N S \_I, \quad M O G \times P I L S, M O V N S \times P I L S$ and GMOVNS $\times$ PILS.

\section{Conclusions}

This work addressed the resource-constrained project scheduling problem with precedence relations as a multi-objective optimization problem, having two optimization criteria that were tackled: the makespan minimization and the minimization of the total weighted start time of the activities.

To solve the problem, five algorithms were implemented: MOG, MOVNS, MOG using VNS as local search, denominated GMOVNS; MOVNS with intensification procedure based on Ottoni et al. [24], denominated MOVNS_I; and PILS.

The algorithms were tested in 160 instances adapted from literature, and compared using four multi-objective performance measures: distance, hypervolume, epsilon and error ratio. Based on the results attained from the computational experiments, we can see that all algorithms were computationally efficient, obtaining sets of non-dominated solutions in an acceptable time, and three conclusions were obtained: first, the MOVNS_I has shown to be superior than the other algorithms on the majority of instances, regarding the distance metrics; second, the GMOVNS is superior regarding the hypervolume indicator and the epsilon metric; and third, the algorithm PILS is superior regarding the error ratio. Statistical experiments were conducted and have revealed that there is a significant difference between some proposed algorithms concerning the distance, epsilon, and error ratio metrics. However, significant difference between the proposed algorithms with respect to hypervolume indicator was not observed.

\section{Acknowledgments}

The authors would like to thank the UFOP, UFV and FAPEMIG for their support and incentive in the development of this work.

\section{References}

[1] Oguz O, Bala H. A comparative study of computational procedures for the resource constrained project scheduling problem. Eur J Oper Res 1994;72:406-16.

[2] Garey MR, Jonhson DS. Computers and intractability: a guide to the theory of NP-completeness. New York: W.H. Freeman and Company; 1979. 
[3] Thomas PR, Salhi S. A Tabu search approach for the resource constrained project scheduling problem. J Heuristics 1998;4:123-39.

[4] Slowinski R. Multi-objective project scheduling under multiple-category resource constraints. Advances in project scheduling. Amsterdam: Elsevier; 1989.

[5] Martínez-Irano M, Herrero JM, Sanchis J, Blasco X, Garcia-Nieto S. Applied Pareto multi-objective optimization by stochastic solvers. Eng Appl Artif Intell 2009;22:455-65.

[6] Ballestín F, Blanco R. Theoretical and practical fundamentals for multiobjective optimization in resource-constrained project scheduling problems. Comput Oper Res 2011;38:51-62.

[7] Jones DF, Mirrazavi SK, Tamiz M. Multi-objective metaheuristics: an overview of the current state-of-art. Eur J Oper Res 2002;137:1-19.

[8] Hansen MP. Tabu search for multi-objective optimization: MOTS. In: Proceedings of the 13th international conference on multiple criteria decision making. University of Cape Town; 1997. p. 6-10.

[9] Czyzak P, Jaszkiewicz A. Pareto simulated annealing - a metaheuristic technique for multiple-objective combinatorial optimization. J Multi-Criteria Decis Anal 1998;7:34-47.

[10] Deb K, Agrawal S, Pratap A, Meyarivan T. A fast elitist nondominated sorting genetic algorithm for multi-objective optimization: NSGA-II. In: Schoenauer M, editor. Parallel problem solving from nature (PPSN VI). Berlin, Germany: Springer; 2000. p. 849-58.

[11] Zitzler E, Laumanns M, Thiele L. SPEA2: improving the strength Pareto evolutionary algorithm. Zurich, Switzerland: Computer Engineering and Networks Laboratory (TIK); 2001.

[12] Viana A, Sousa JP. Using metaheuristics in multi-objective resource constrained project scheduling. Eur J Oper Res 2000;120:359-74.

[13] Kazemi FS, Tavakkoli-Moghaddan R. Solving a multi-objective multi-mode resource-constrained project scheduling with discounted cash flows. In: Proceedings of the 6th international management conference. Tehran, Iran; 2008.

[14] Abbasi B, Shadrokh S, Arkat J. Bi-objective resource-constrained project scheduling with robustness and makespan criteria. Appl Math Comput 2006;180:146-52.

[15] Al-Fawzan MA, Haouari M. A bi-objective model for robust resourceconstrained project scheduling. Int J Prod Econ 2005;96:175-87.

[16] Aboutalebi RS, Najafi AA, Ghorashi B. Solving multi-mode resource-constrained project scheduling problem using two multi-objective evolutionary algorithms. Afr J Bus Manage 2012;6(11):4057-65.

[17] Reynolds AP, Iglesia B. A multi-objective GRASP for partial classification. Soft Comput 2009;13:227-43.

[18] Geiger MJ. Randomized variable neighborhood search for multi-objective optimization. 4th EU/ME: design and evaluation of advanced hybrid metaheuristics; 2008. p. 34-42.

[19] Geiger MJ. Foundations of the Pareto iterated local search metaheuristic. In: Proceedings of the 18th MCDM. Chania, Greece; June 2006.

[20] Arroyo JEC, Vieira PS, Vianna DS. A GRASP algorithm for the multi-criteria minimum spanning tree problem. Ann Oper Res 2008;159(1):125-33.

[21] Minella G, Ruiz R, Ciavotta MA. Review and evaluation of multi-objective algorithms for the flowshop scheduling problem. INFORMS J Comput 2010;20:451.

[22] Oliveira Junior PL, Arroyo JEC, Souza VAA. Heurísticas GRASP e ILS para o Problema No-wait Flowshop Scheduling Multiobjetivo. In: Proceedings of the 17th Simpósio Brasileiro de Pesquisa Operacional. Bento Gonçalves, Brasil; 2010 .

[23] Arroyo JEC, Ottoni RS, Oliveira AP. Multi-objective variable neighborhood search algorithms for a single machine scheduling problem with distinct due windows. Electron Notes Theor Comput Sci 2011;281:5-19.

[24] Ottoni RS, Arroyo JEC, Santos AG, Algoritmo VNS. Multiobjetivo para um Problema de Programação de Tarefas em uma Máquina com Janelas de Entrega. In: Proceedings of the 18th Simpósio Brasileiro de Pesquisa Operacional. Ubatuba, Brasil; 2011.

[25] Koné O, Artigues C, Lopez P, Mongeau M. Event-based MILP models for resource-constrained project scheduling problems. Comput Oper Res 2009;38:3-13.

[26] Kolisch R. Serial and parallel resource-constrained project scheduling methods revisited: theory and computation. Eur J Oper Res 1996;90:320-33.

[27] Kelley JE. The critical method: resources planning and scheduling. Industria scheduling. New Jersey: Prentice-Hall; 1963; 347-65.

[28] Feo TA, Resende MGC. Greedy randomized adaptive search procedures. J Glob Optim 1995;40:109-33.

[29] Mladenovic N, Hansen P. Variable neighborhood search. Comput Oper Res 1997:24:1097-100.

[30] Lourenço HR, Martin OC, Stüzle T. Iterated local search. In: Glover F, Kochenberger GA, editors. Handbook of metaheuristics. Dordrecht, Netherlands: Kluwer Academic; 2003. p. 320-53.

[31] Deiranlou M, Jolai FA. New efficient genetic algorithm for project scheduling under resource constraint. World Appl Sci J 2009;7:987-97.

[32] Agarwal A, Colak S, Erenguc SA. Neurogenetic approach for the resourceconstrained project scheduling problem. Comput Oper Res 2011:38:44-50.

[33] Kolisch R, Sprecher A. PSPLib - a project scheduling problem library. Eur J Oper Res 1996;96:205-16.

[34] PSPLib. 〈http:/129.187.106.231/psplib/〉.

[35] Hansen M, Jaszkieweiz A. Evaluating the quality of approximations to the nondominated set. Department of Mathematical Modeling, Technical University of Denmark; 1998 ([technical report]).

[36] Veldhuizen D. Multi-objective evolutionary algorithms: classifications, analyses and new innovations. Dayton, Ohio: School of Engineering of the Air Force Institute of Technology; 1999 ([Ph.D. thesis]).

[37] Zitzler E, Ded K, Thiele L. Comparison of multi-objective evolutionary algorithms: empirical results. Evol Comput 2000;8:173-95.

[38] Deb K, Jain S. Running performance metrics for evolutionary multi-objective algorithms. Kanpur Genetic Algorithms Laboratory, Indian Institute of Technology Kanpur, India; 2002 ([technical report]).

[39] Fonseca C, Knowles J, Thiele L, Zitzler EA. Tutorial on the performance assessment of stochastic multi-objective optimizers. In: Proceedings of the 3th international conference on evolutionary multi-criterion optimization (EMO). Guanajuato, Mexico; 2005.

[40] Coello CA, Lamont GB. Applications of multi-objective evolutionary algorithms. world scientific printers. Singapore: Pte Ltd.; 2004

[41] Montgomery DC. Design and analysis of experiments. 7th ed.. New York: John Wiley and Sons; 2009.

[42] Kulinskaya E, Staudte RG, Gao A. Power approximations in testing for unequal means in a one-way ANOVA weighted for unequal variances. Commun Stat 2003;32(12):2353-71.

[43] Montgomery DC, Runger CR. Estatística Aplicada e Probabilidade para Engenheiros. 5th ed.. Brasil: LTC; 2012. 